\title{
Redesigning Informed Consent: Enhancing Risk Communication Through a Novel Risk Visualization Tool
}

\author{
Thomas Arthur ${ }^{\mathrm{a}, \mathrm{b}}$, c, Alfred Phillips ${ }^{\mathrm{a}}$, Zanna Franks ${ }^{\mathrm{a}}$, Stacey Pearce ${ }^{\mathrm{b}}$
}

\begin{abstract}
Background: The communication of risk is a difficult task, with evidence to suggest that doctors perform poorly in the attempt to convey it. Consent forms should be designed to assist in informing patients of these risks, and encourage their comprehension. The authors present a pilot study into a novel risk visualization tool (RVT) for the informed consent process, which provides probabilistic and severity information in a single graphical form.
\end{abstract}

Methods: This is a prospective, randomized study of adult volunteers. One hundred volunteers were taken through a mock consent process for a laparoscopic appendicectomy, with either the RVT or standard institutional form.

Results: There was greater recall of surgical risks in the group that utilized the RVT $(4.94 / 8.0,61 \%)$ compared to the standard consent form $(3.52 / 8.0,44 \%)(\mathrm{P} \leq 0.005)$. Participants who were given the RVT were also more likely to recall the most common risk and the most severe risk. Participants who were given the RVT also reported greater satisfaction with the informed consent process than those patients given the standard consent form.

Conclusion: The results of the study suggest that a patient's ability to comprehend the risks of a surgical procedure and satisfaction with the consenting process can be improved with the use of the RVT.

Keywords: Consent; Surgery; Communication

\section{Introduction}

Informed consent for a surgical procedure involves the dis-

\footnotetext{
Manuscript accepted for publication August 19, 2015

aGold Coast University Hospital, Gold Coast, QLD, 4215, Australia ${ }^{\mathrm{b}} \mathrm{S}$ chool of Medicine, Griffith University, Gold Coast, QLD, 4215, Australia ${ }^{\mathrm{c} C}$ Corresponding Author: Thomas Arthur, Department of Surgery, Gold Coast University Hospital, 1 Hospital Blvd, Southport, QLD 4215, Australia.

Email: Thomas.arthur@uqconnect.edu.au
}

doi: http://dx.doi.org/10.14740/jcs275w closure, and subsequent comprehension, of a number of risks. Consent forms should be designed to inform patients of these risks, and encourage their comprehension. Evidence suggests in practice this is not that case. Paasche-Orlow et al found a significant number of institutional review boards of medical schools in the US failed to produce consent forms for clinical trials that meet their own minimum readability standards [1]. If readability suffers, can we assume that some patients are not even reading their consent forms? This is certainly what some studies have suggested, with $60-70 \%$ of patients either "admitting to not reading the consent form" or not reading it "carefully" [2]. Such forms disempower and dissociate patients from the decision making process.

We present a pilot study into a novel risk visualization tool (RVT) for the informed consent process, which provides probabilistic and severity information in a single graphical format. The objective of this study was to assess whether the RVT was able to improve the comprehension and retention of the surgical risks of a laparoscopic appendicectomy. Doing so serves to improve patient safety and satisfaction by enhancing understanding of the risks involved in undergoing a surgical procedure.

\section{Material and Methods}

Ethics approval for this study was obtained by our institutions human research review committee. Adult volunteers were recruited from public areas of a major tertiary hospital. Subjects were approached to be part of the study. Those who have had previous abdominal surgery were precluded from participation in the trial.

Participants were taken through a mock informed consent for a laparoscopic appendicectomy. Participants were randomized to either receiving the RVT or the standard consent form in an alternate fashion (Fig. 1 and 2). The consent form was presented to the subject, and the information was read through twice. Subjects were then given a distraction task, to diminish their immediate recall ability. The consent form was withdrawn, they were presented with a generic picture of an abdomen and asked five questions related to the picture.

Subjects were asked a series of questions to assess their recall of the surgical risks (Table 1), and their answers were recorded. 


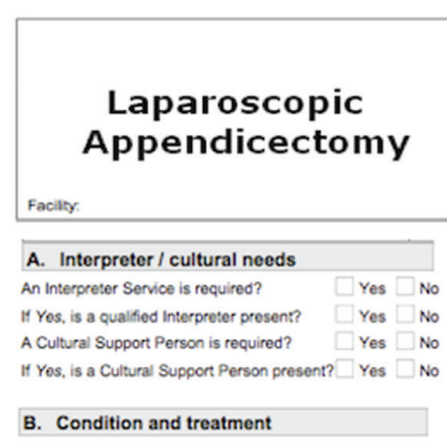

The doctor has explained to you that you have the following condition: (Doctor to document in patients own words)

Appendicitis-1...

The condition requires the following procedure/treatment/investigation. (Doctor to document - include site and/or side where relevant to the procedure.

Laparoscopic Appendicectomy-..-...-..--

A laparoscopic appendicectomy is a keyhole procedure to remove a person's appendix. It is often used to treat appendicitis. A camera will be inserted into the abdomen. The operation involves three small incisions in the abdomen, the abdomen is expanded with gas and a camera and instruments inserted to remove the appendix.

C. Risks of this procedure

As with any operation there are a number of risks to be aware of. It's important to remember that complications are uncommon, but should be taken into account before undergoing ay operation.

They include but are not limited to the following.

- Infection can occur, requiring antibiotics. Collections may occur in the abdominal cavity, requiring surgical treatment.

- Not all operations can be performed with keyhole surgery. Your procedure may need to be performed with a larger cut,

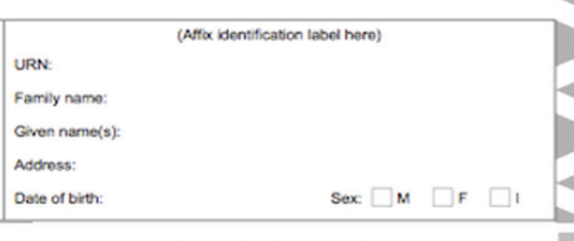

- A weakness in the wound can occur, with development of a hernia.

- Deep bleeding form the wound, or deep inside the abdomen may occur. This may require fluid replacement, blood transfusion, or further surgery.

- Adhesions (or scar tissue) may form and cause a bowel obstruction. This may require further surgery.

- Damage to other organs, such as bladder or bowel, which may need further surgery.

- Blood may clot in the leg causing pain and swelling (DVT). In rare cases part of the clot may break off and go to the lungs.

- Heart attack or stroke could occur due to strain on the heart. Death, although very rare, may result from any procedure.

\section{Significant risks and procedure options}

(Doctor to document in the space provided. Continue on the medical record if necessary.)

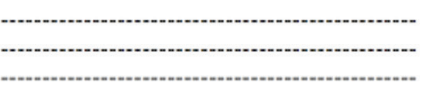

E. Risks of not having this procedure

(Doctor to document in the space provided. Continue on the medical record if necessary.)

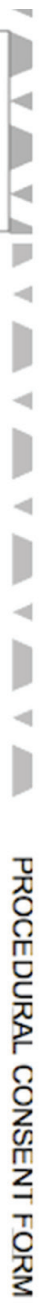

Figure 1. Standard institutional consent form.

Subjects were then asked to appraise three statements via a five-point Likert scale. Participants utilizing the RVT were asked to respond to the questions in Table $2 \mathrm{a}$.

Participants utilizing the standard consent form were

\section{Table 1. Questions on Frequency and Severity of Risks}

Q1 What are the risks or potential adverse events that can occur when undergoing a laparoscopic appendicectomy?

Q2 Which of these adverse events are the most likely to occur?

Q3 Which of these adverse events are the most serious? asked to respond to the questions in Table $2 \mathrm{~b}$.

Answers were collated and underwent statistical assessment by an independent biostatistician using SPSS v21.

\section{Results}

The means for all response variables among the two study subgroups were statistically compared using independent samples $t$-tests. Since these response variables deviate slightly from the assumptions of normality, we confirmed our results by performing non-parametric comparison of medians. A P-value of 


\section{LAPAROSCOPIC APPENDICECTOMY}

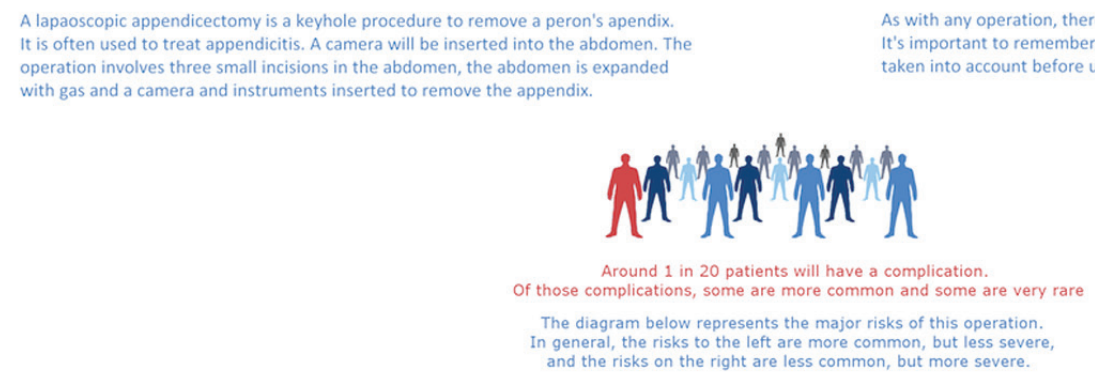

COMPLICATIONS

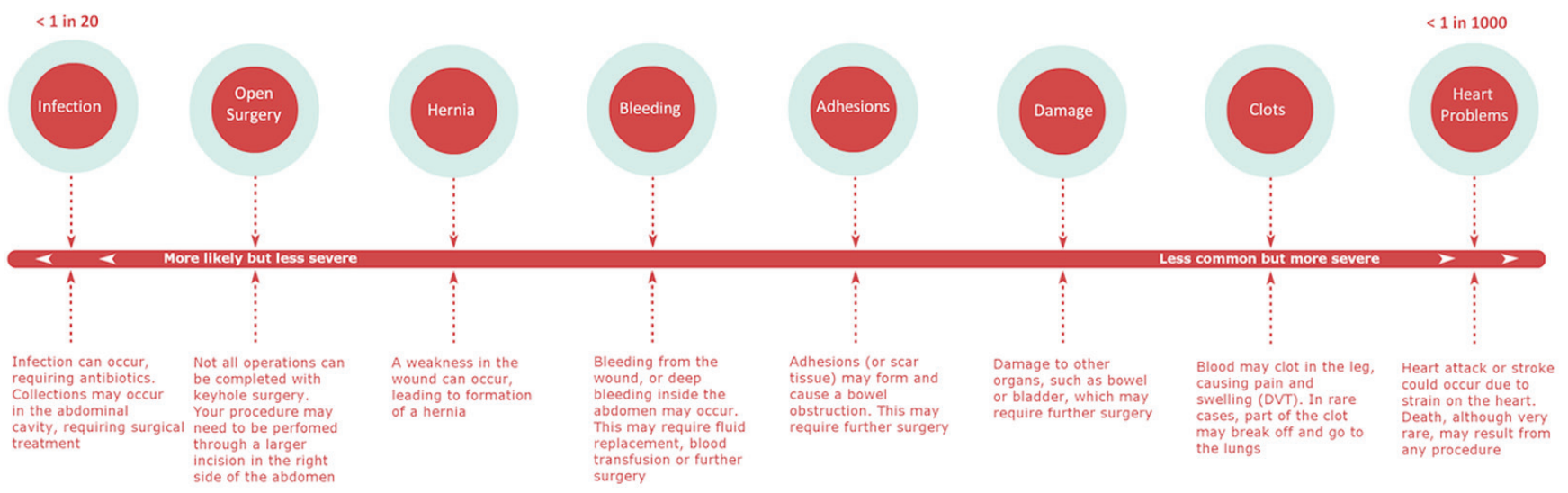

Figure 2. Risk visualization tool.

$<0.05$ was considered statistically significant.

\section{Subject profile}

Thirty-nine percent of respondents were aged $18-24$ years, $24 \%$ between 25 and 35 years, $22 \%$ between 35 and 55 years, and $15 \%$ over 55 years.

\section{Recall}

Participants who received the RVT were able to recall an aver- age of 4.94 of the eight risks mentioned on the consent form. Those who received the standard consent form were only able to recall 3.52 of the eight risks on the consent form. This difference was statistically significant $(\mathrm{P}=0.005)$.

\section{Most common and most severe risk}

Forty-two of the participants given the RVT were able to recall "infection" as the most common risk, compared to 34 participants who utilized the standard form $(\mathrm{P}=0.05)$. Forty-six of the participants given the RVT were able to recall "heart problems" as the most severe risk, compared to 43 who utilized the

Table 2. Statements on Self-Reported Comprehension Assessed via 5-Point Likert Scale

$\begin{array}{ll}\text { a. RVT } & \text { b. Control }\end{array}$

Q1. The use of a "risk visualization tool" made learning about the procedure and its risks easier to understand.

Q1. The informed consent process made learning about the procedure and its risks easy to understand.

Q2. The use of a "risk visualization tool" made learning about Q2. The informed consent process made learning about the procedure and its the procedure and its risks easier to remember. risks easier to remember.

Q3. If you were to undergo a laparoscopic appendicectomy, the use of a "risk visualization tool" would make it easier to Q3. If you were to undergo a laparoscopic appendicectomy, the use of an extra visual information such as this (show RVT to participant) would make understand and remember the risks of having the procedure. it easier to understand and remember the risks of having the procedure. 
standard form. This was not statistically significant $(\mathrm{P}=0.36)$.

\section{Self-reported understanding and integration}

Subjects given the RVT were more likely to report that the risks were easier to understand (RVT 4.5/5, non-RVT 3.62/5, $\mathrm{P} \leq 0.001$ ) and easier to remember (RVT 4.34/5, non-RVT $3.24 / 5, \mathrm{P} \leq 0.001$ ), than if given the standard institutional form.

\section{Utility of the RVT}

Participants shown the RVT after undergoing the mock consent with the standard institutional form rated the use of "extra visual information" highly as a possible adjunct to aid in the consenting process (4.56/5). Patients who solely used the RVT also rated the instrument highly $(4.46 / 5)$.

\section{Time}

Time taken to consent participants utilizing the standard consent form was $3 \mathrm{~min} 50 \mathrm{~s}$. Time taken to consent participants utilizing the RVT was $4 \mathrm{~min}$ and $5 \mathrm{~s}$. This difference was not statistically significant $(\mathrm{P}=0.1)$.

\section{Discussion}

The risks in the RVT are presented in a visual form, in what may be described as an information graphic. Visual aids have been used as an adjunct to the presentation of data in medicine for many years - the ubiquitous pie chart was first popularized by Florence Nightingale to describe causes of mortality of soldiers during the Crimean War. The usefulness of graphical presentation of information has been explored previously in making informed consent a more informed process. Methods explored include graphs and tables [3], pictograms [4], videotapes [5], and interactive multimedia programs [6]. Altering the style and substance of consent forms has shown mixed results in improving patient comprehension [7].

The RVT differs from previous attempts in that it combines the graphical representation of risk and the consent form in one paper instrument. Videotapes and interactive web programs in particular require specialized equipment to consent the patient. The RVT requires no more resources, nor time to use, compared to a standard consent form. This is of obvious importance in time-sensitive fields such as surgery, where one has little time to take a patient through a complex interactive multimedia programme or video.

Participants who were taken through the mock consent process with the RVT were able to recall more risks than those who used the standard consent form. This is despite the fact that the core information on each consent form was almost entirely identical. Humans have a remarkable capacity for remembering visual information. Standing et al showed 10,000 images to individuals for a few seconds each, and found they were able to recall which pictures they had previously seen with $84 \%$ accuracy [8]. Visual recognition has been proven to be "systematically superior" to auditory recognition [9]. Despite the evidence for the superiority of visual recognition and memory, standard consent forms often persist to be text-based and presented in a plain fashion.

The RVT differed from the standard consent form not only visually, but also by the inclusion of probabilistic and severity information. It is possible that this in itself may have improved participants' recall of risk. The levels-of-processing effect described by Craik and Lockhart in 1979 proposes that the provision of context may enhance the encoding of memory [10]. The comprehension of each risk may have been facilitated in providing context related to its relative frequency and severity.

The instrument not only resulted in an improvement in the recall of risks, but individuals also self-reported greater integration of the information provided to them when utilizing the RVT. This suggests that the instrument improves patient satisfaction with the informed consent process when utilizing the RVT, as compared to the standard form.

One problem in formulating the RVT was prioritizing whether a risk was "more likely" or "more severe" than another. This is a difficult task, as reported complication rates vary widely, as will the severity of a complication. Infection was placed at the most common and least severe end of the scale; however, a severe infection can be just as life-threatening as a heart attack. Published data on complication rates were used to assist in ascertaining the frequency of each complication $[11,12]$. Severity of a complication was rated subjectively by the authors. An individual's assessment of whether a complication is more severe than another will depend on their own value system. The RVT included a caveat to advise that the frequency/severity scale was to be utilized in general terms, rather than prescriptively.

The results of this pilot study would have been strengthened if the health literacy levels of the participants was ascertained and controlled for. The distraction task could have been an opportunity to assess this, with a scoring of the participants' answers to simple health questions serving as a surrogate for their health literacy. Younger volunteers (18 - 24) are overrepresented in the sample; however, the peak incidence of appendicitis is between the ages of 10 and 30 years [13].

The RVT created is intended as an alternative to the traditional consent form. Participants who utilized the RVT had greater recall of risks, and greater self-reported uptake of information, than those who utilized the standard consent form. It is the aim of the authors to further explore the utility of the RVT in a hospital inpatient setting. The findings of this study suggest that the RVT may be a valuable adjunct to the decision making process for patients undergoing surgical operations.

\section{Grant Support}

None.

\section{Financial Disclosures}

None. 


\section{References}

1. Paasche-Orlow MK, Taylor HA, Brancati FL. Readability standards for informed-consent forms as compared with actual readability. N Engl J Med. 2003;348(8):721-726.

2. Lavelle-Jones C, Byrne DJ, Rice P, Cuschieri A. Factors affecting quality of informed consent. BMJ. 1993;306(6882):885-890.

3. Kurz-Milcke E, Gigerenzer G, Martignon L. Transparency in risk communication: graphical and analog tools. Ann N Y Acad Sci. 2008;1128:18-28.

4. Gett RM, Cooray AR, Gold D, Danta M. Evaluating informed consent for colonoscopy. Surg Laparosc Endosc Percutan Tech. 2014;24(4):345-352.

5. Weston J, Hannah M, Downes J. Evaluating the benefits of a patient information video during the informed consent process. Patient Educ Couns. 1997;30(3):239-245.

6. Batuyong ED, Jowett AJ, Wickramasinghe N, Beischer AD. Using multimedia to enhance the consent process for bunion correction surgery. ANZ J Surg. 2014;84(4):249-254.

7. Nishimura A, Carey J, Erwin PJ, Tilburt JC, Murad MH, McCormick JB. Improving understanding in the research informed consent process: a systematic review of 54 interventions tested in randomized control trials. BMC Med Ethics. 2013;14:28.

8. Standing L. Learning 10,000 pictures. Q J Exp Psychol. 1973;25(2):207-222.

9. Cohen MA, Horowitz TS, Wolfe JM. Auditory recognition memory is inferior to visual recognition memory. Proc Natl Acad Sci U S A. 2009;106(14):6008-6010.

10. Craik FIM, Lockhart RS. Levels of processing. A framework for memory research. J Verb Learn Verb Beh. 1972;11:671-684.

11. Li X, Zhang J, Sang L, Zhang W, Chu Z, Liu Y. Laparoscopic versus conventional appendectomy--a meta-analysis of randomized controlled trials. BMC Gastroenterol. 2010;10:129.

12. Tsao KJ, St Peter SD, Valusek PA, Keckler SJ, Sharp S, Holcomb GW, 3rd, Snyder CL, et al. Adhesive small bowel obstruction after appendectomy in children: comparison between the laparoscopic and open approach. J Pediatr Surg. 2007;42(6):939-942; discussion 942.

13. Schwartz SI. Principles of surgery. New York. McGraw Hill. 1994;1307-1318. Appendix. 